\title{
RBP2 induces stem-like cancer cells by promoting EMT and is a prognostic marker for renal cell carcinoma
}

\author{
Dahai Zhou ${ }^{1,2}$, Vinodh Kannappan ${ }^{3}$, Xiang $\mathrm{Chen}^{4}$, Jingqin $\mathrm{Li}^{2}$, Xuefeng Leng ${ }^{2}$, Jinping Zhang ${ }^{2}$ \\ and Shiying Xuan ${ }^{1,5}$
}

Renal cell carcinoma (RCC), one of the most common kidney cancers, has a poor prognosis. Epithelial to mesenchymal transition (EMT) is a hallmark of carcinoma invasion and metastasis. Several studies have examined the molecular regulation of EMT, but the relationship between histone demethylases and EMT is little understood. In this study, we investigated the role of retinoblastoma-binding protein-2 (RBP2), a histone demethylase that is highly expressed in RCC and is positively correlated with poor RCC prognosis in the regulation of EMT. We found that ectopic overexpression of RBP2 can induce cancer stem cell-like (CSC) phenotypes through EMT in RCC cells by converting them to a more mesenchymal phenotype. This results in increased resistance to apoptosis, which leads to enhanced tumor growth in xenograft models. Together, our data show that RBP2 is an epigenetic regulator that has an important role in the initiation of CSC phenotypes through EMT, leading to tumor progression. RBP2 is also a novel biomolecule for RCC diagnosis, and prognosis and may be a therapeutic target.

Experimental \& Molecular Medicine (2016) 48, e238; doi:10.1038/emm.2016.37; published online 10 June 2016

\section{INTRODUCTION}

Renal cell carcinoma (RCC) is an intricate set of diseases whose incidence has risen steadly throughout the world. In 2013, RCC was diagnosed in more than 350000 people worldwide, and it is associated with more than 140000 deaths annually. ${ }^{1}$ Despite increased incidence rates, there has been no significant improvements in relative survival rates over the past 30 years. ${ }^{2,3}$ RCC is a collective term that refers to a group of cancers that originate in the epithelium of renal tubules. It comprises three main histopathological entities, among which, clear cell RCC is the dominant histology, accounting for $\sim 65 \%$ of reported cases, followed by papillary and chromophobe RCC, which account for $\sim 15-20 \%$ and $5 \%$ of reported cases, respectively. Rarer subtypes make up the remainder of RCC cases, including collecting duct, mucinous tubular, spindle cell, renal medullary and MiTF-TFE translocation carcinomas., ${ }^{4,5}$ Several major genomic and mechanistic discoveries, including identification of several new rare subtypes of renal cancers, have altered our core understanding of RCC and our knowledge of these cancers is rapidly expanding. ${ }^{5}$
Accumulating evidence in recent years supports the hypothesis that RCC tumors contain a subpopulation of tumor cells called cancer stem cells (CSCs), also known as tumor initiating cells or tumorigenic cells. These cells, exhibit stem cell properties such as self-renewal, tumorsphere formation, the ability to differentiate into heterogeneous populations of cancer cells and can initiate tumors in a xenotransplant system. However, the origin of renal CSCs is still not clear, because of incomplete experimental evidence and contradicting views about the existence of CSCs. ${ }^{6-9}$ Emerging evidence from various types of cancer suggest that the acquisition of epithelial to mesenchymal transition (EMT) and induction of CSCs or cancer stemlike cell phenotypes are interrelated. ${ }^{10-16}$ Studies in other tumor systems indicate that EMT is often activated during cancer invasion and metastasis. ${ }^{17-20}$ EMT is a biological process, in which epithelial cells undergo multiple biochemical changes that enable them to lose their cell-cell basement membrane contacts and their structural polarity (epithelial-like phenotype) to assume a mesenchymal-like phenotype, which includes enhanced migratory potential, invasiveness, increased resistance to apoptosis and high secretion of extracellular

\footnotetext{
${ }^{1}$ College of Medicine and Pharmacy, Ocean University of China, Qingdao, China; ${ }^{2}$ Department of Urology, 401 Hospital of PLA, Qingdao, China; ${ }^{3}$ Research Institute in Healthcare Science, Faculty of Science \& Engineering, University of Wolverhampton, Wolverhampton, UK; ${ }^{4}$ Tangdu Hospital, Xi'an, China and ${ }^{5}$ Qingdao Municipal Hospital, Qingdao, China

Correspondence: Professor S Xuan, College of Medicine and Pharmacy, Ocean University of China, 5 Yushan Road, Qingdao, Shandong 266071, China. E-mail: dxyqdslyy@sina.com

Received 12 November 2015; revised 30 December 2015; accepted 6 January 2016
} 
matrix (ECM) components. ${ }^{21-23}$ Although EMT and CSCs have a vital role in tumor metastasis, resistance and relapse, on their own they cannot explain the various cellular events that occur in tumor progression. In particular, the significance of EMT signaling in regulating the stemness of CSCs is still not fully understood, ${ }^{13,15,16,24}$ and careful evaluation of these two concepts has led researchers to explore a promising link between EMT and the CSC phenotype. ${ }^{24}$ However, few studies have examined EMT-induced CSCs in RCC. Studies of EMT in RCC have focused on the expression of a single EMT gene or limited sets of EMT-related genes, and mostly at the protein level by immunohistochemical analyses. Few quantitative gene expression studies at the mRNA level have been performed to assess EMT in RCC. ${ }^{25-27}$

Mounting evidence suggest that the activation of EMT signaling and its associated genes are governed by epigenetic modifications. Histone methylation on specific lysine residues is an epigenetic mechanism that regulates gene expression by making the promoter region of a gene accessible or inaccessible to transcription factors (TFs), and aberrant methylation leads to tumorigenesis. ${ }^{28-31}$ Histone lysine methylation, like many other epigenetic mechanisms, can be reversed through the action of demethylases, among which the KDM5/JARID1 family of histone demethylases has a role in the removal of di- and tri- methyl marks from lysines on histone $\mathrm{H} 3$. Retinoblastoma-binding protein-2 (RBP2), also known as KDM5A or JARID1A, one of the four members of the JARID1 protein family, is highly conserved and redundant demethylation activities. RBP2, was originally isolated as a binding partner of retinoblastoma protein $(\mathrm{pRB})^{31-33}$ and subsequently shown to be involved in transcriptional regulation through its ability to directly bind target DNA through an AT-rich interaction domain. ${ }^{31}$ The connection between RBP2 and abnormal cell growth was first identified because of its function as a binding partner of the tumor suppressor $\mathrm{pRB}$, which is frequently inactivated, either directly or indirectly, in many cancers. ${ }^{34}$ The ability of pRB to promote differentiation and senescence is strongly correlated with its ability to bind to RBP2. ${ }^{35}$ Because H3K4 trimethylation is often associated with the promoter regions of actively transcribed genes, RBP2 was thought to exert its function through transcriptional repression of those genes. However, subsequent studies showed that RBP2 can also activate gene expression through less defined mechanisms. It has also been shown that, using the AT-rich interaction domain binding sequence, RBP2 can interact with many oncogenic proteins, such as p107, Myc, TBP, HDACs, nuclear receptors and RBPJ. ${ }^{32,36-38}$

RBP2 has been shown to have an important role in breast and prostate cancer progression by interacting with nuclear receptors and recruiting additional factors important for nuclear receptor-mediated transcription, resulting in enhanced target gene expression. ${ }^{37} \mathrm{RBP} 2$ is an essential component of the Notch/RBP-J repressor complex and is shown to suppress Notch signaling through histone demethylation, which results in suppression of Notch-induced tumorigenesis. ${ }^{39}$ RBP2 is also found to be overexpressed in gastric cancer ${ }^{40}$. In both gastric and cervical cancer, RBP2 binds to the promoter regions of cyclin-dependent kinase inhibitors p16, p21 and p27 to hinder their expression and reduce the senescence of cancer cells. ${ }^{40}$ Similarly, RBP2 binds to the promoters of p27, cyclin D1 and integrin $\beta 1$ to mediate lung cancer proliferation and metastasis. ${ }^{41}$ RBP2 has also been shown to form a fusion protein with the nuclear pore complex protein NUP98 in acute myeloid leukemia patients. Further studies using murine models revealed that the RBP2-NUP98 complex inhibits demethylase activity and enhances the expression of lineagespecific TFs. This in turn halts the hematopoietic differentiation that results in acute myeloid leukemia. ${ }^{42}$ A recent study indicated that high RBP2 expression could promote CSC phenotypes and increase chemoresistance by changing chromatin structure. ${ }^{43}$ Abrogation of RBP2 resulted in loss of the 'stemness' property from mouse embryonic stem cells in vitro and inhibited cell growth, induced senescence and differentiation. Moreover, loss of RBP2 significantly inhibited tumorigenesis in a mouse cancer model, suggesting that RBP2 might be a promising prognostic factor and an attractive therapeutic target in multiple cancer types. ${ }^{44}$ In this study, we analyzed the role of RBP2 in inducing EMT in RCC, resulting in stem cell-like phenotypes that are often associated with poor prognosis.

\section{MATERIALS AND METHODS}

\section{Ethics statement}

All experimental procedures were approved by the ethics committee of 401 Hospital, Qingdao, China. Patient information and clinical samples were obtained after informed written consent had been provided, according to an established protocol.

\section{Patients and tumor specimens}

Human RCC specimens were obtained from 121 patients with RCC in 401 Hospital, Qingdao, China from 2010 to 2014. The diagnosis of RCC and the pathological grade were confirmed by histological examination, and no patient had undergone preoperative radiotherapy or chemotherapy. All samples were collected immediately after surgery and stored in formalin or frozen at $-80^{\circ} \mathrm{C}$ until use. Overall survival was measured using the follow-up data available from the hospital database, and any lost follow-up was regarded as death caused by RCC, according to the 2002 Union for International Cancer Control standard.

\section{Cell culture and gene overexpression}

SKRC and SN12C human RCC cell lines were obtained from the Chinese Academy of Science (Shanghai, China) and cultured in Roswell Park Memorial Institute-1640 (RPMI 1640) (Gibco, Carlsbad, CA, USA) supplemented with $10 \%(\mathrm{v} / \mathrm{v})$ heat-inactivated fetal bovine serum (Gibco), $100 \mathrm{U} \mathrm{ml}^{-1}$ penicillin and $100 \mathrm{mg} \mathrm{ml}^{-1}$ streptomycin (Gibco). Cells were incubated at $37^{\circ} \mathrm{C}$ in a humidified atmosphere $\left(5 \% \mathrm{CO}_{2}\right)$. For gene overexpression, the SKRC and SN12C cell lines were cultured overnight in six-well plates $\left(1.0 \times 10^{6}\right.$ cells per well), followed by transfection with $5 \mu \mathrm{g} \mathrm{ml}^{-1}$ pcDNA-RBP2 plasmid (Genepharma, Shanghai, China), or empty plasmid using $5 \mu \mathrm{l}$ of Lipofectamine 2000 reagent (Invitrogen, Carlsbad, CA, USA). The cells were incubated for $24 \mathrm{~h}$ and positive clones were isolated by G418 (Sigma, St Louis, MO, USA) selection using $600 \mu \mathrm{g} \mathrm{ml}^{-1} \mathrm{G} 418$. 
Protein extraction, SDS-polyacrylamide gel electrophoresis and western blot

Total cellular proteins were extracted using radio-immunoprecipitation analysis lysis buffer, and protein concentration was measured using a standard Bradford assay protocol. Equal amounts of proteins were separated by SDS-polyacrylamide gel electrophoresis, and then transferred onto nitrocellulose membranes. The membranes were then blocked with $5 \%$ non-fat milk for $1 \mathrm{~h}$, followed by incubation with primary antibodies at $4{ }^{\circ} \mathrm{C}$ overnight. Antibodies for RBP2, BCL-2, BAX and proliferating cell nuclear antigen were purchased from Abcam (Cambridge, MA, USA), and antibodies against E-cadherin, $\mathrm{N}$-cadherin, vimentin, SOX2, OCT4 and NANOG were purchased from Cell Signaling Technology (USA). Subsequently, the blots were washed and probed with horseradish peroxidaseconjugated secondary antibodies (Santa Cruz Biotechnology, CA, USA) for $2 \mathrm{~h}$, and complexes were detected by enhanced chemiluminescence (PerkinElmer Life Sciences). Finally, the blots were washed and probed with an antibody against tubulin (Santa Cruz Biotechnology), which was used as a loading control.

\section{MTT assay}

The MTT [3- (4,5-dimethylthiazol-2yl)-2,5-diphenyltetrazolium bromide] cell viability assay was used to measure cell survival, thereby conversely measuring any cellular events leading to apoptosis or necrosis, which would affect the cell proliferation rate. Cells $\left(5 \times 10^{3}\right.$ cells per well) were seeded in a 96-well plate and incubated at $37^{\circ} \mathrm{C}$ for $0-3$ days. Then, the cells were incubated with medium containing $5 \mathrm{mg} \mathrm{ml}^{-1}$ MTT (Sigma) for $2 \mathrm{~h}$ at $37^{\circ} \mathrm{C}$, followed by solubilization of the resulting insoluble formazan crystals with $200 \mu$ l of dimethyl sulfoxide. Absorbance at $570 \mathrm{~nm}$ was read using a microplate reader (Bio-Rad, Hercules, CA, USA).

\section{Flow cytometric analysis of CD133 and CD44 expression}

The cells to be analyzed were trypsinized into single cells, and $\sim 2.5 \times 10^{5}$ cells were counted and incubated in flow buffer ( $2 \%$ fetal bovine serum in PBS) containing CD133 or CD44 antibodies (BD Biosciences, San Diego, CA, USA) for $20 \mathrm{~min}$ at $4{ }^{\circ} \mathrm{C}$. Any unbound antibodies were washed off with flow buffer, and the cells were rapidly analyzed in fluorescence-activated cell sorting (FACS) scan (BD Biosciences). Approximately 10000 events were collected, and any increase or decrease in the expression of CD133 and CD44 was analyzed using Cell Quest Pro software (BD Biosciences). All experiments were performed in triplicate and each set was repeated three times.

\section{ALDEFLUOR-ALDH assay}

An ALDEFLUOR kit (StemCell Technologies, USA) was used to determine any increase in aldehyde dehydrogenase (ALDH) enzymatic activity, which is a functional marker of CSCs. Cells to be analyzed $\left(2.5 \times 10^{5}\right.$ cells $)$ were suspended in ALDEFLUOR assay buffer containing ALDH substrate and incubated for $30 \mathrm{~min}$ at $37^{\circ} \mathrm{C}$ according to the manufacturer's instructions. As a negative control, an aliquot of cells was incubated with diethylaminobenzaldehyde (DEAB), a specific inhibitor of ALDH. The amount of intracellularfluorescence was measured by flow cytometry using an FACS scan and analyzed using Cell Quest Pro software.

\section{Total RNA extraction and PCR with reverse transcription and real-time PCR}

Cells were lysed using TRIzol reagent (Invitrogen), and total RNA was isolated according to the manufacturer's protocol. The isolated RNA was then reverse-transcribed with an iScript cDNA Synthesis kit (Bio-Rad Laboratories). The cDNA obtained was used to perform semi-quantitative PCR using a PCR with reverse transcription kit (Promega, Madison, WI, USA) and real-time PCR using iQ SYBR Green Supermix (Bio-Rad) in a Bio-Rad iQ5 thermal cycling system (Bio-Rad) following the instructions from the supplier. The following primer sequences were used:

Oct-4F: 5'-TCAGGTTGGACTGGGCCTAGT-3' R: 5'-GGAGGTTC CCTCTGAGTTGCTT- $3^{\prime}$

Sox2 F: 5'- GAGGGCTGGACTGCGAACT-3', R: 5'-TTTGCACCC CTCCCAATTC-3'

Nanog F: 5'-GAAATCCCTTCCCTCGCCATC-3', R: 5'-CTCAGTA GCAGACCCTTGTAAGC-3

Twist $\mathbf{F}$ 5'-GCGCTGCGGAAGATCATC-3', $\mathbf{R}$ 5'-GGTCTGAATC TTGCTCAGCTTGT- $3^{\prime}$.

Snail F5'-CCTGGGTGCCCTCAAGATGC-3', R5'-GAGCAGGGAC ATTCGGGAGA-3'

GAPDH F5'-CAAGGTCATCCATGACAACTTCG-3', R5'-GTCCA CCACCCTGTTGCTGTAG-3'

\section{Tumorsphere formation assay}

To determine the role of RBP2 in enhancing CSC characteristics, we evaluated the ability of clones to form floating tumorspheres in non-adhesive conditions. We plated $\sim 1 \times 10^{3}$ cells per well in 24-well ultra-low attachment plates (Corning Inc., Corning, NY, USA) in a serum-free DMEM/F12 supplemented with 2\% B27 and 1\% N2 supplement (Life Technologies, Invitrogen), $20 \mathrm{ng} \mathrm{ml}^{-1}$ human EGF, $10 \mathrm{ng} \mathrm{ml}^{-1}$ human bFGF and $5 \mu \mathrm{g} \mathrm{ml}^{-1}$ insulin. After 14 days of culture at $37^{\circ} \mathrm{C}$, plates were inspected for tumorsphere formation. The spheres formed were dissociated using a non-enzymatic solution (Sigma) and were re-plated with fewer cells (1000) per well for another 14 days, after which the number and size of the spheres formed were quantified using an inverted microscope.

\section{Chromatin-immunoprecipitation (ChIP) assay}

Mock or RBP2 overexpressing cells were cultured and chemically crosslinked with $1 \%$ formaldehyde for $30 \mathrm{~min}$ at $37^{\circ} \mathrm{C}$. Cells were lysed and sonicated with 4-mm tip probe for three cycles, $10 \mathrm{~s}$ each, to ensure that the crosslinked DNA was sheared and solubilized. The chromatin extract was incubated with $2 \mu \mathrm{g}$ of RBP2 antibody (Abcam) at $4{ }^{\circ} \mathrm{C}$ overnight for precipitation. The DNA-RBP2 protein-antibody complex was eluted and reverse crosslinked with $20 \mu \mathrm{l}$ of $5 \mathrm{M} \mathrm{NaCl}$. Anti-Rabbit IgG was used as a negative control and genomic DNA was used as input DNA. The presence of E-cadherin and Bcl2 promoter containing a RBP2-binding site in immunoprecipitated DNA was identified by PCR amplification and resolved on 1\% agarose DNA gels. The following primers were used: E-cadherin - forward: $5^{\prime}$-CAAA GTGGGCACAGATGGTGT G- $3^{\prime}$ and reverse: $5^{\prime}$-CTGCTTGGATTCC AGAAACGG-3'. Bcl2—forward: 5'-CTAGTCTAGAGCCTCAGGGAA CAGAATGATCAG-3'and reverse:5'-CTAGTCTAGAAAGCGTCCAC GTTCTTCATTG3'.

\section{In vivo tumor growth}

Four-week-old BALB/C nude mice were purchased from the Shanghai Experimental Animal Center, Chinese Academy of Sciences (Shanghai, China). The mice were housed under pathogen-free conditions according to the animal care guidelines of Ocean University of China. The animal experiments were reviewed and approved by the Ethical Committee of Ocean University of China. A total of $5 \times 10^{6}$ cells from log phase cultures of mock or RBP2 overexpressing SKRC-39 cells 
were injected subcutaneously into the flank regions of each animal. Tumor volume was calculated using the following formula: $V=$ $(L \times W 2) \times 0.5$, where $L$ is the length and $W$ is the width of the tumor. The xenograft size was observed every week for 4 weeks. The animals were killed after 4 weeks, and the tumors were removed and weighed.

\section{Immunohistochemistry}

Paraffin-embedded sections of mice tumor tissues were stained according to standard protocols with primary antibody against Ki67 (Abcam) followed by staining with biotinylated anti-mouse secondary antibody. After washing any unbound antibodies, the sections were incubated with a buffer containing HRP-conjugated streptavidin followed by addition of substrate solution containing peroxidise. The slides were mounted with 3,3'-diaminobenzidine and visualized with a Leica light field microscope.

\section{Terminal deoxyribonucleotidyltransferase-mediated dUTP nick end labeling (TUNEL) assay}

In situ TUNEL staining was performed using an ApoTag peroxidase in situ apoptosis detection kit. The paraffin-embedded tumor tissues

a

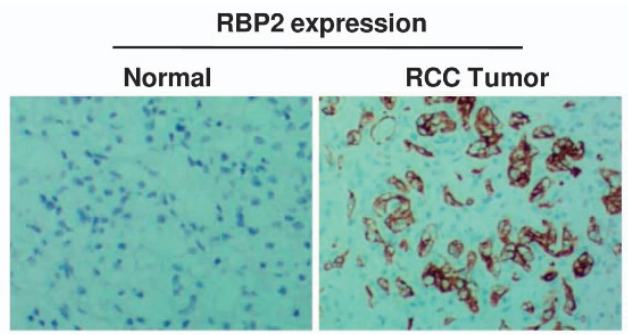

b
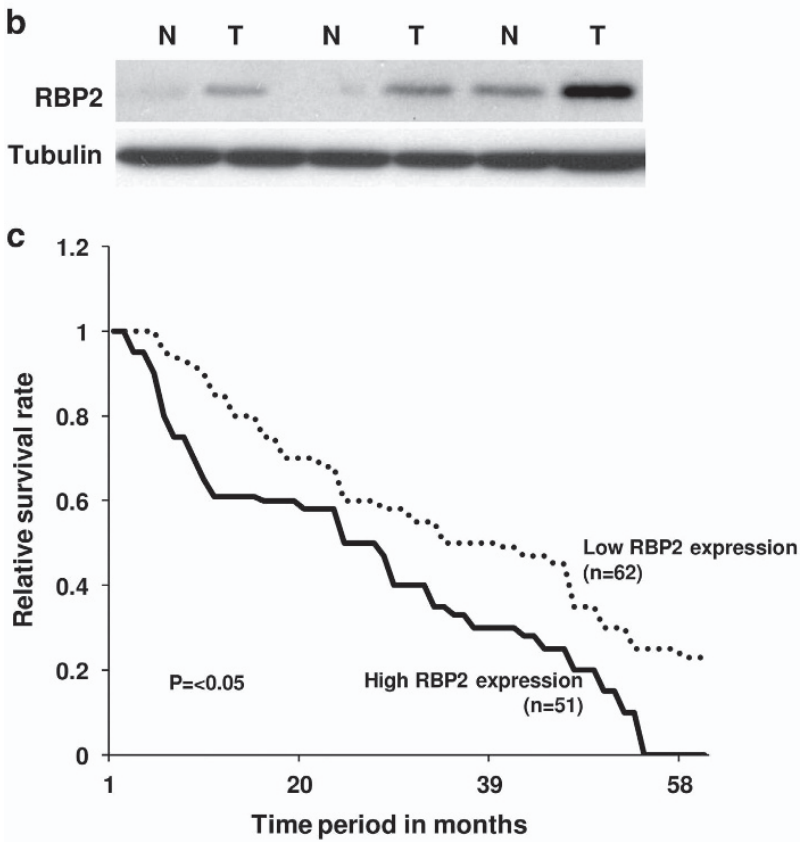

Figure $1 \mathrm{RBP} 2$ is frequently upregulated in RCC and is closely associated with poor survival rates of RCC patients. (a) Representative immunohistochemical images of high RBP2 expression in RCC tissue specimens $(\times 100)$. (b) Western blot results of elevated RBP2 protein levels in RCC samples in comparison with normal tissue samples. (c) Kaplan-Meier survival curves of RCC patients according to the status of RBP2 expression. sections were deparaffinized and rehydrated, followed by the addition of $200 \mu$ proteinase $\mathrm{K}$ solution $\left(10 \mu \mathrm{g} \mathrm{ml}^{-1}\right)$ and stained according to the manufacturer's protocol. Briefly, the sections were incubated with terminal deoxynucleotidyltransferase (TdT) enzyme followed by stop/wash buffer and addition of anti-digoxigenin. The sections were then incubated with peroxidase substrate and $\mathrm{ABC}$ reagent. Finally, the slides were mounted with 3,3'-diaminobenzidine and visualized under a light microscope.

\section{Statistical analysis}

Values were expressed as the mean \pm s.d. $P<0.05$ was considered significant. SPSS13.0 (St Jose, CA, USA) was used for statistical analysis. Discontinuous data were analyzed by $\chi^{2}$-test or an exact probability test. The Kaplan-Meier method was used to analyze the total survival rate and disease-specific survival rate. A log-rank test was used to determine the prognostic value.

\section{RESULTS}

RBP2 is frequently upregulated in RCC and is positively correlated with differentiation status and poor prognosis To investigate the expression of RBP2 in RCC, we collected tumor tissues and adjacent non-tumor tissues from 121 RCC patients. The expression status of RBP2 in patient samples was determined by immunohistochemistry, which showed that RBP2 protein was significantly upregulated in RCC compared with normal tissues (Figure 1a). To confirm the increased expression of RBP2, the protein levels of RBP2 in RCC specimens were also analyzed by western blotting. We found that the protein levels of RBP2 were also higher in tumor specimens than in normal tissues (Figure 1b).

The differentiation status of a primary tumor provides information about cancer aggressiveness. Poorly differentiated tumors are usually highly progressive and possess greater metastatic potential. To determine whether RBP2 expression is correlated with RCC differentiation status and progression, we conducted a statistical analysis of RBP2 expression levels (low or high) and the tumor differentiation status (good, moderate or poor differentiation) in 121 RCC samples (Table 1). We found that, among the 22 (out of 121) well-differentiated RCC tumors, 18 samples (81.8\%) had low expression of RBP2. Among the 47 RCC samples with moderate differentiation, more than half of the samples (55\%) had high RBP2 expression and 45\% had low RBP2 expression. Interestingly, among the 52 samples that had poor differentiation status, 39 (75\%) had high RBP2 expression, indicating a strong inverse relationship between RBP2 expression and tumor differentiation status $(P<0.05)$. However, there was no significant relationship between RBP2 expression and other aspects, such as age or gender (Table 1).

To determine whether high RBP2 expression level and the associated low tumor differentiation status resulted in a poor survival rate, we investigated the prognosis and follow-up data for all 121 RCC patients from the database at the 401 Hospital, Qingdao. Remarkably, the analysis revealed that the survival rate of patients with high expression of RBP2 was significantly lower than that of the patients with low RBP2 expression. $(P<0.05$, Figure 1c). 
Table 1 Expression levels of RBP2 in RCC patients and its relation with clinical features and tumor differentiation status in primary RCC specimens

\begin{tabular}{|c|c|c|c|c|}
\hline \multirow[b]{2}{*}{ Groups } & \multirow[b]{2}{*}{ Total } & \multicolumn{2}{|c|}{ RBP2 expression } & \multirow[b]{2}{*}{ P-valu } \\
\hline & & $0-1$ & $2-3$ & \\
\hline \multicolumn{5}{|l|}{ Age (years) } \\
\hline$<60$ & 42 & $20(47.6 \%)$ & $22(53.4 \%)$ & $>0.05$ \\
\hline$>60$ & 79 & $42(53.2 \%)$ & $37(46.8 \%)$ & \\
\hline \multicolumn{5}{|l|}{ Gender } \\
\hline Male & 98 & $47(48.0 \%)$ & $51(52.0 \%)$ & $>0.05$ \\
\hline Female & 23 & $12(54.5 \%)$ & $11(45.5 \%)$ & \\
\hline \multicolumn{5}{|c|}{ Tumor differentiation } \\
\hline Good & 22 & $18(81.8 \%)$ & $4(18.2 \%)$ & $<0.05$ \\
\hline Moderate & 47 & $21(44.7 \%)$ & $26(55.3 \%)$ & \\
\hline Poor & 52 & $13(25.0 \%)$ & $39(75.0 \%)$ & \\
\hline
\end{tabular}

Abbreviations: RBP2, retinoblastoma-binding protein-2; RCC, renal cell carcinoma.

\section{Exogenous overexpression of RBP2 promotes EMT in RCC} cell lines

The above data suggested a strong link between RCC progression and RBP2 expression. Studies from various tumor types in the past decade have indicated that EMT is often activated during cancer progression and metastasis. To investigate the potential role of RBP2 in RCC progression through the activation of EMT, we overexpressed RBP2 exogenously in two RCC cell lines: SKRC-39 and SN12C. We confirmed an increased expression of RBP2 in cells transfected with pcDNARBP2 plasmid by western blotting, and the expression levels were found to be stable over serial passages. By contrast, the cells transfected with empty vector alone had very low or undetectable levels of RBP2 expression, with levels very similar to that of parental cells (Figure 2a). EMT is a complex biological process characterized by the loss of epithelial markers and subsequent acquisition of mesenchymal markers. We investigated the effect of RBP2 overexpression on protein expression levels of the epithelial marker E-cadherin and the mesenchymal markers $\mathrm{N}$-cadherin and vimentin. As shown in Figure $2 \mathrm{~b}$, cells overexpressing RBP2 exhibited a significant downregulation of E-cadherin and a dramatic upregulation of $\mathrm{N}$-cadherin and vimentin in comparison with cells expressing only the empty vector. This indicated that EMT is activated following RBP2 overexpression. To further confirm this, we analyzed the effect of overexpressing RBP2 on the expression of the key EMT TFs Snail and Twist. We found that there is a dramatic increase in Twist and Snail expression in cells overexpressing RBP2 when compared with the control cells (Figure 2c). To confirm the direct involvement of RBP2 in inducing EMT, we determined the ability of RBP2 to bind directly to the promoter of E-cadherin using a ChIP assay. Our results from both SKRC39 and SN12C cells showed that exogenous upregulation of RBP2 leads to the direct binding of functional RBP2 to the promoter region of the E-cadherin gene, resulting in to the suppression of E-cadherin and eventual EMT (Figure 2d). These molecular changes indicate an apparent transition of the RBP2-expressing SKRC-39 and SN12C cells from an epithelial to mesenchymal status.

\section{RBP2 mediates EMT-generated stem-like cells}

Recent studies in various tumor types have highlighted a link between EMT and acquisition of CSC properties including the presence of CSC markers, tumorigenicity, ability to redifferentiate into a tumor and ability to form spheres in culture. To determine whether RBP2-induced EMT can lead to stem cell phenotypes, we performed FACS analysis to identify expression of CSC markers in RBP2-transfected cells. We observed that cells transfected with RBP2 exhibited a dramatic increase in the expression of CD133 and CD44 cell-surface CSC markers (Figures $3 \mathrm{a}$ and $\mathrm{b}$ ). In addition, we used the ALDEFLUOR assay to measure any increase in ALDH activity, a hallmark characteristic of CSCs. The ALDH activity was significantly higher in cells with high RBP2 expression, confirming that these cells exhibit CSC characteristics (Figure 3c). To further confirm the CSC nature of these cells, we analyzed the mRNA and protein levels of three important embryonic TFs: Sox2, Oct4 and Nanog. Real-time PCR and western blot results showed a significant increase in all the above mentioned embryonic TFs in RBP2 expressing cells (Figures 3d and e). The expression of such early transcriptional regulators is necessary for the maintenance of pluripotency in stem cells. Tumorsphere formation assays are widely used to assess the self-renewing ability of cells with stem cell characteristics. As shown in Figure 3f, the RBP2-expressing RCC cells displayed an increased ability to form tumorspheres in comparison with the control cells. The spheres formed in RBP2 expressing cells were larger and formed at a faster rate than the control cells (Figure 3g). This suggests that ectopic overexpression of RBP2 induces EMT in both RCC cell lines and generates mesenchymal cells with stem cell-like phenotypes.

\section{Exogenous overexpression of RBP2 promotes resistance to apoptosis}

To determine whether RBP2 is directly involved in augmentation of cell proliferation and can support tumor growth, we performed a cell proliferation assay using standard MTT analysis of the RBP2 overexpressing RCC cells. In addition to the MTT assay, we analyzed the DNA contents and cell cycle profile of these cells by FACS. From the MTT analysis, we observed a very low level increase, (but not a statistically significant increase, $P>0.05)$ in the proliferation rate of cells overexpressing RBP2 in comparison with control cells of both cell lines (Figure 4a). However, we did not observe any marked differences between the cell cycle profiles of these cells (Figure $4 \mathrm{~b}$ ). We confirmed the above results by detecting the protein expression levels of proliferating cell nuclear antigen by western blotting, which showed no remarkable difference between the control and RBP2-transfected cells (Figure 4d). Both results indicated that an elevated expression of RBP2 does not affect the cell proliferation rate significantly. However, the 


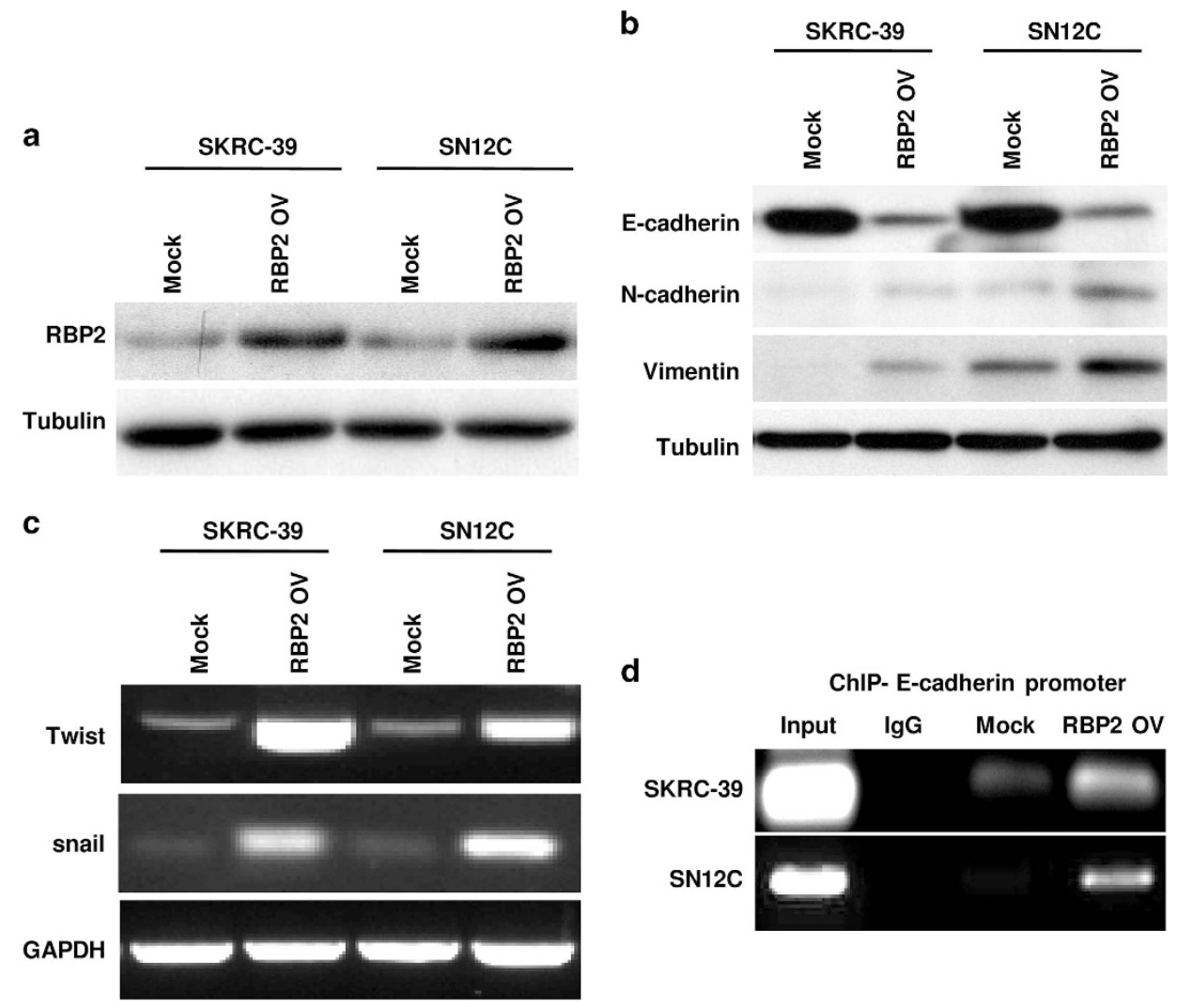

Figure 2 Ectopic overexpression of RBP2 in two RCC cell lines SKRC-39 and SN12C, induced an EMT program. (a) Western blotting assessment of elevated expression of RBP2 protein in RCC cell lines stably transfected with pCDNA-RBP2 in comparison with cells transfected with empty vector alone. (b) Cells with high RBP2 show reduced expression of the epithelial marker E-cadherin and elevated levels of the mesenchymal markers N-cadherin and vimentin. (c) mRNA expression of EMT factors analyzed by RT-PCR. (d) The ChIP assay shows that RBP2 is a DNA binding protein that can directly bind to the promoter sequence of E-cadherin DNA. RT-PCR, PCR with reverse transcription.

DNA content profile revealed a significant reduction in the sub-G0 population of RBP2 overexpressed cells in both RCC cell lines, demonstrating that apoptosis is inhibited in these cells. Resistance to apoptosis is a characteristic feature of mesenchymal or stem cell-like phenotypes, and leads to poor prognosis in patients. When quantified using FACS, the apoptotic population in RBP2 expressing cells was three- to fourfold less than that of control cells (Figure 4c). We also examined whether RBP2 overexpression is associated with alteration in the expression of apoptosis regulating proteins Bax and Bcl2. As shown in Figure 4d, we detected a downregulation of the proapoptotic protein Bax and an upregulation of the anti-apoptoic protein $\mathrm{Bcl} 2$ after transfection of RCC cells with RBP2, in comparison with the cells transfected with empty vector. Furthermore, our ChIP analysis revealed that RBP2 can directly bind to the $\mathrm{Bcl} 2$ promoter in order to alter the $\mathrm{Bcl} 2$ : Bax ratio (Figure 4e). To our knowledge, this is the first study to show direct involvement of RBP2 in suppressing apoptosis in cancer cells.

\section{RBP2 enhances tumorigenicity of RCC in vivo}

To investigate the role of RBP2 in RCC tumorigenicity in vivo, SKRC-39 RCC cells with exogenous overexpression of RBP2 were injected into nude mice. Control group mice were injected with the same cells transfected with the empty vector only. The results from tumor volume analysis indicated that animals injected with cells expressing RBP2 exhibited rapid tumor progression, resulting in bigger tumors than the control group at the end of 4 weeks (Figure 5a). Analysis of the tumor weight from both groups confirmed this result; tumors from the RBP2 overexpressed group were nearly twice as heavy as the control group tumors (Figure 5b). Tissue sections from these tumors subjected to immunohistochemistry staining with Ki67 showed no significant changes in proliferation of the tumors in vivo(Figure 5c). However, TUNEL staining showed a significant reduction in apoptotic cells in tumors isolated from mice injected with RBP2 overexpressing cells in comparison with that of control group tumors (Figure $5 \mathrm{~d}$ ). These results are directly in line with our in vitro findings. Although we did not observe any change in proliferation rates, the animals injected with RBP2 overexpressing cells developed aggressive and rapidly progressing tumors, which underlines the important role of RBP2 in tumor progression and poor prognosis in patients with RCC.

\section{DISCUSSION}

RCC is considered one of the gravest cancers because of its high recurrence and mortality rate. Because it is extremely 


\section{a CD133 expression}
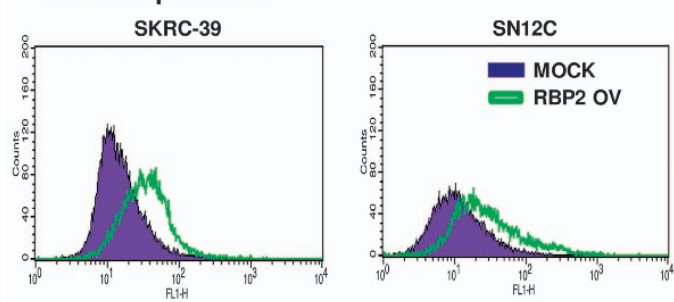

b CD44 expression
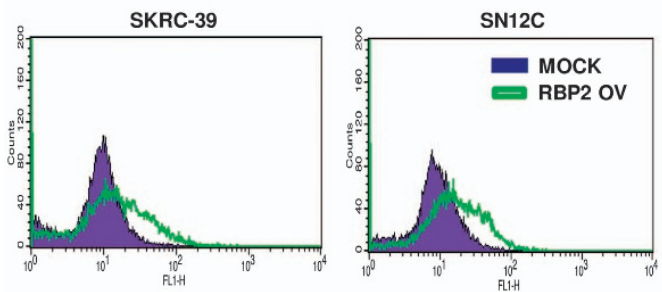

C ALDH Activity
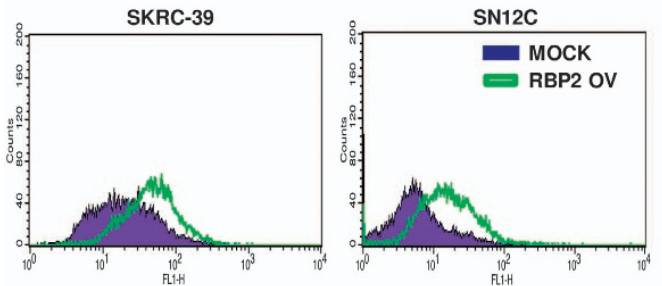
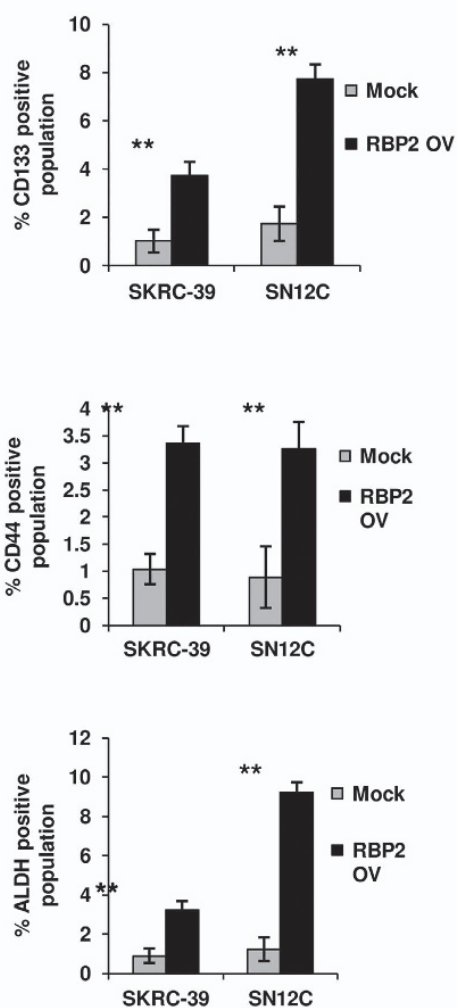

SN12C
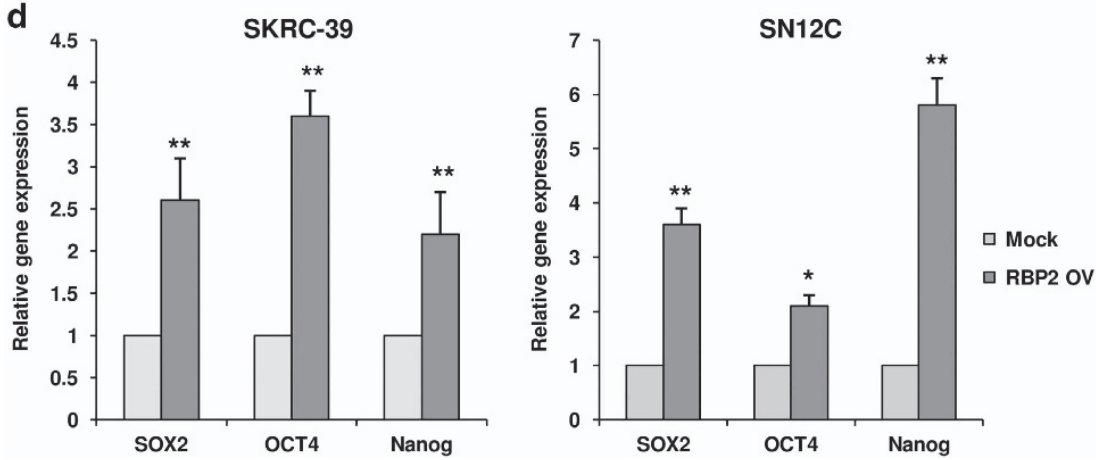

Figure 3 RBP2-nduced EMT-generated stem cell-like cells. (a) FACS analysis shows an increase in CSC markers ALDH, (b) CD133 and (c) CD44 in RBP2-transfected cells. Bar charts indicate the percentage of cells positive for CSC markers $(* * P<0.01 ; n=9)($ d) quantitative real-time PCR showed high mRNA expression of CSC TFs Sox2, Oct4 and Nanog in RBP2-transfected cells $\left({ }^{*} P<0.05 ;{ }^{* *} P<0.01 ; n=3\right.$ ). (e) Western blot shows increased protein levels of Sox2, Oct4 and Nanog in RBP2-transfected cells. (f) Representative microscopy images of tumorsphere formation $(\times 100)$ and $(\mathrm{g})$ quantification of tumorspheres formed from three experiments $\left({ }^{*} P<0.05\right)$.

resistant to chemotherapy, radiation or immunotherapy, complete responses are very rare and the prognosis is poor. RCC progression is a complex, multi-factorial process that involves epigenetic modifications that have yet to be fully elucidated. It is becoming increasingly evident that the CSC population in tumors cannot be eradicated by conventional treatment strategies, which leads to tumor recurrence and poor survival. CSC theory has recently been proposed in connection in RCC, with the identification of putative renal CSCs that may have a relevant role in determining its progression and prognosis. Although targeting CSCs could be an attractive strategy to improve the clinical outcome, it is not easy to achieve because they display dynamic stemness properties dependingon the tumor microenvironment. ${ }^{12,24}$ In line with this, it has been shown in many tumors that CSCs are often induced by EMT where the resulting mesenchymal phenotype displays CSC markers. ${ }^{1-16}$ EMT is a highly dynamic, multistep process requiring the destabilization of cell-cell interactions brought about by activation of TFs, expression of specific cell-surface proteins, reorganization of cytoskeletal proteins, production of extracellular matrix degrading enzymes and other epigenetic modifications. ${ }^{24}$

Histone methylation is a key epigenetic mechanism in the control of gene expression, cell cycle and DNA repair, and any 


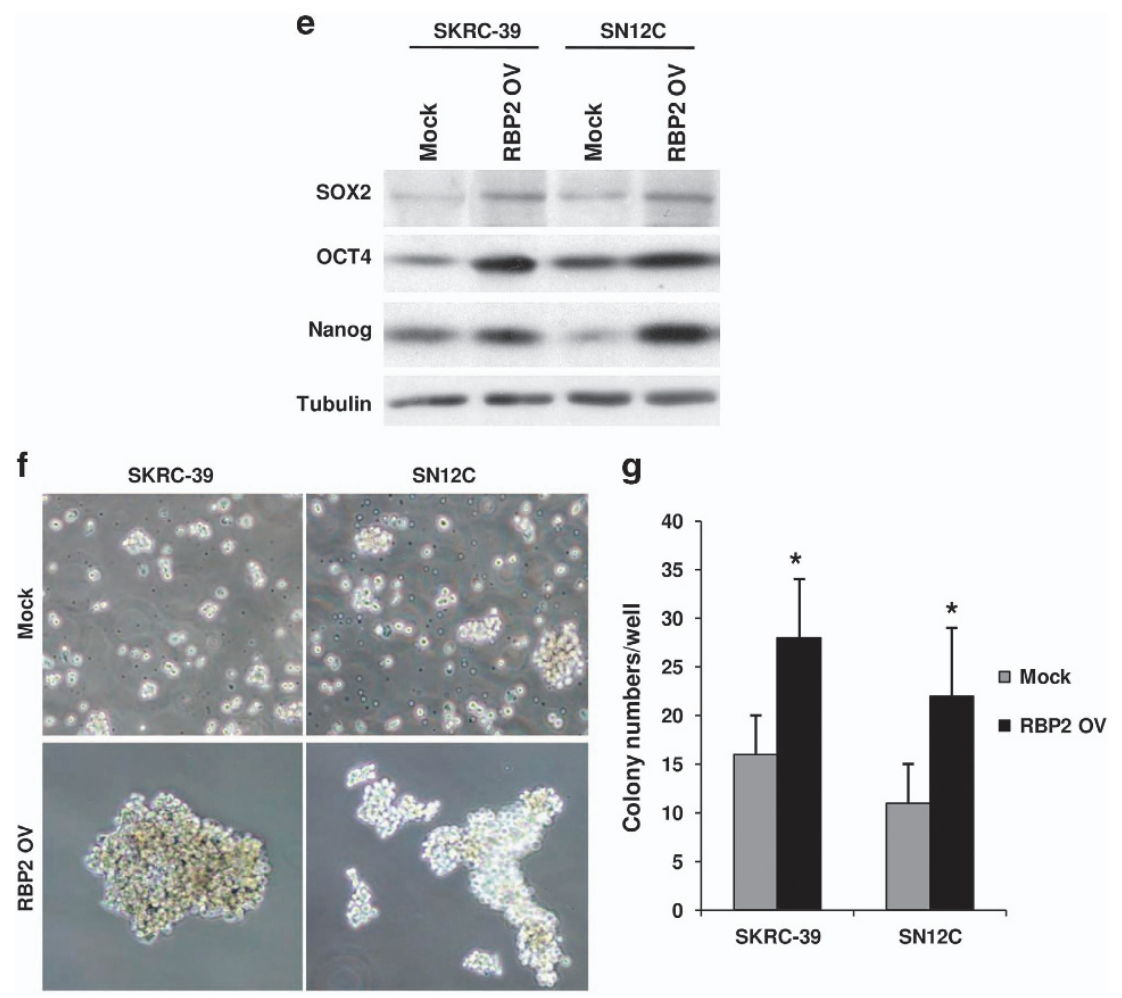

Figure 3 Continued.

aberration in this process is related to tumorigenicity. ${ }^{31}$ In the present study, we found that RBP2, an H3K4 demethylase, may have a key role in RCC progression, leading to poor clinical outcomes. RBP2 has been implicated as an oncoprotein in various cancer types, including melanoma, gastric, breast, lung and ovarian cancers. ${ }^{45}$ In this study, we determined that RBP2 is frequently upregulated in RCC and has a high correlation with poor tumor differentiation and poor survival rates in RCC patients, suggesting that RBP2 could be a critical epigenetic switch in RCC progression. Because RBP2 is a molecule related to the control of gene expression and is dynamically affected by epigenetic modifications, we sought to connect RBP2 expression to EMT and stemness of RCC leading to poor prognosis. We found that RBP2 overexpression in RCC cell lines can readily transform the cells into a more mesenchymal phenotype. This is evident from the downregulation of epithelial genes, such as E-cadherin, and the upregulation of mesenchymal genes, such as $\mathrm{N}$-cadherin and vimentin, in addition to the elevated expression of EMT TFs Twist and Snail. Snail is a well-known TF that suppresses E-cadherin expression, a key molecule in the maintenance of the tight intercellular junctions of cancer epithelium. Our findings also demonstrate that RBP2 has a direct role in repression of E-cadherin protein expression through the direct binding of RBP2 to the promoter region of the E-cadherin gene. It has previously been shown in non-small cell lung cancer that RBP2 can directly bring about a change in $\mathrm{N}$-cadherin protein expression by activating the Akt signaling pathway. 46
We also observed that EMT induced by RBP2 overexpression can lead to RCC cell lines with stem-like cell populations. We found that RBP2-transfected cells also displayed a panel of CSC markers such as ALDH, CD133 and CD44 and acquired the ability to form tumorspheres. The expression of CD44 is closely related to TGF $\beta$-mediated EMT and is known to be an important molecule in the inter-conversion between the epithelial and mesenchymal state of cells. ${ }^{12,47}$ In addition to CSC surface markers, we observed that embryonic TFs such as Oct4, Nanog and Sox2 were also upregulated at the protein level. These findings imply that CSCs do not essentially originate from transformed normal stem cells, as they could also de-differentiate from fully differentiated cells that had acquired self-renewal ability and became malignant. ${ }^{6,11}$ CSCs exhibit quiescence, a resistant nature and a lack of apoptotic signals. ${ }^{15}$ Although we did not observe any quiescent nature or a decrease in cell proliferation, we did notice that RBP2 expression directly resulted in reduced apoptotic cell numbers. Contrary to the prevalent view that RBP2 can repress the expression of a gene only through histone demethylation, it has been shown that RBP2 has diverse functions in gene regulation including activation of gene expression (Benevolenskaya et al. ${ }^{35}$ ; Wang et al. ${ }^{42}$ ). Here we showed that RBP2 promotes Bcl-2 expression via direct binding to the $\mathrm{Bcl}-2$ promoter region. Therefore, our data suggest that higher expression of RBP2 may contribute to cell survival by promotion of CSCs through EMT and alteration of apoptotic events, especially in the mitochondria-mediated pathways, through upregulation of Bcl-2 expression in RCC. 

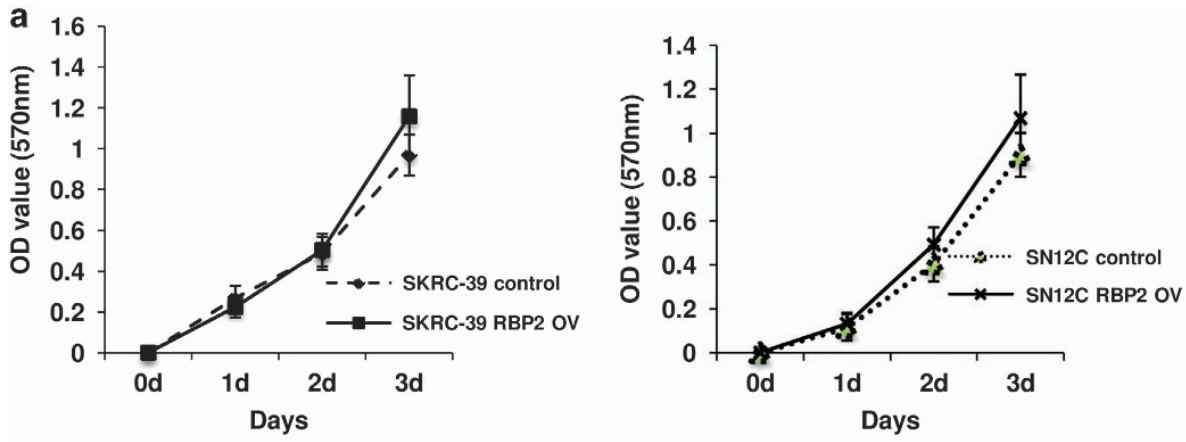

b
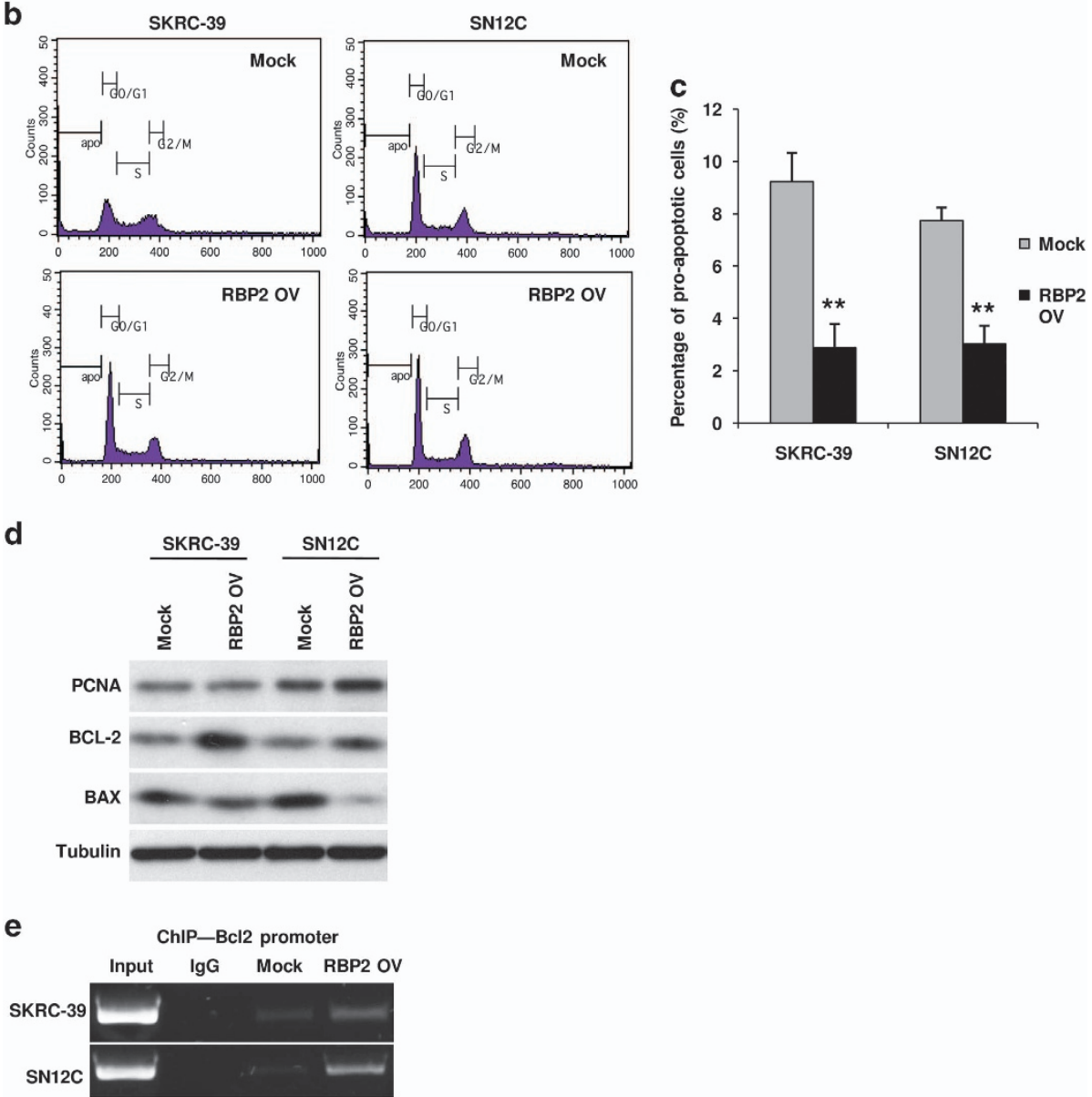

Figure 4 Exogenous overexpression of RBP2 did not affect cell proliferation but promoted resistance to apoptosis in RCC cell lines SKRC-39 and SN12C. (a) MTT assay shows no change in proliferation rates of RBP2-transfected cells. (b) FACS DNA content analysis for the cell cycle profile of RBP2 expressing cells and control cells. (c) Quantification of apoptotic cells from DNA contents shows a reduced apoptotic population in RBP2-transfected cells. (d) Western blot analysis of proliferating cell nuclear antigen, Bcl-2 and Bax proteins indicating resistance to apoptosis but no change in proliferation of RBP2-transfected cells. (e) ChIP assay indicates binding of RBP2 to the $\mathrm{Bcl}-2$ promoter.

In recent years, a better understanding of the molecular pathways involved in RCC has led to the recognition of molecular markers that indicate disease status and may predict response to therapy. ${ }^{48}$ EMT is crucial for the progression of malignant tumors; thus, the molecules involved in EMT might be potential prognostic markers in predicting patient outcome. ${ }^{12} \mathrm{~A}$ greater understanding of the underlying biology of RCC could also lead to therapeutic targeting directed toward molecular components of signaling pathways involved in tumor progression. However, very few studies have identified EMT-related molecular markers for RCC patients. Therefore, the present study's implication of RBP2 as a factor that induces EMT and stemness might be a valuable addition to the search for molecular prognostic factors in RCC.

In conclusion, our study provides direct evidence that RBP2 promotes EMT and is involved in RCC cell invasion. We have 

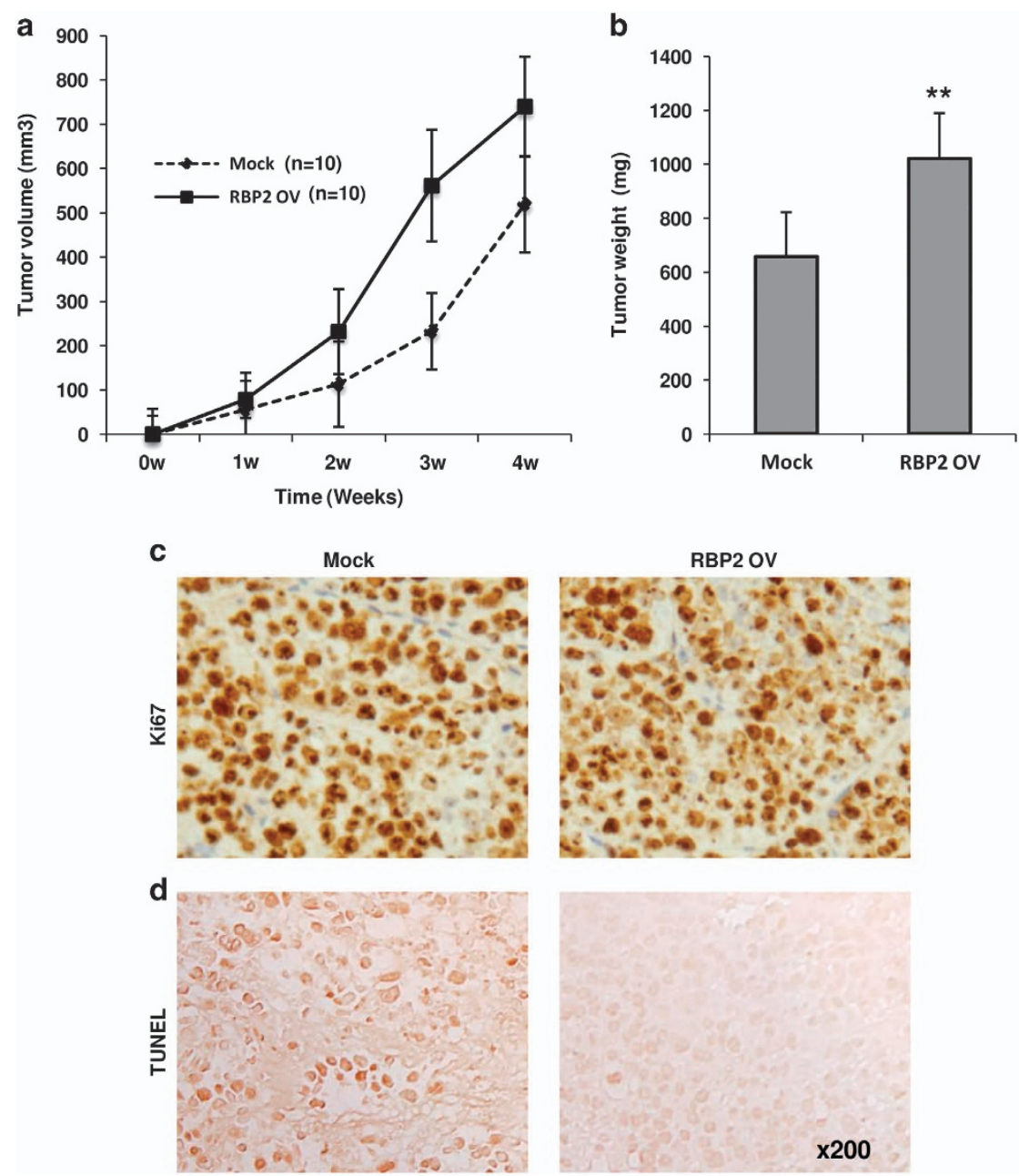

Figure 5 RBP2 enhanced tumorigenicity in vivo. (a) RBP2 overexpressing cells or mock transfected cells were implanted into immunodeficient mice. Tumor volume in $\mathrm{mm}^{3}$ was measured for 4 weeks as indicated and RBP2 overexpressing cells enhanced tumor progression $(P<0.05)$. (b) Upon killing, tumor weights were measured and the RBP2-injected group had bigger tumors $(P<0.01)$. (c) Ki67 and (d) TUNEL staining of the xenografts indicate no change in proliferation rates but fewer apoptotic cells in the RBP2 group than in the control group.

demonstrated that RBP2 has a key role in RCC and therefore serves as a novel therapeutic target and prognostic marker in RCC.

\section{CONFLICT OF INTEREST}

The authors declare no conflict of interest.

1 Ferlay J, Soerjomataram I, Dikshit R, Eser S, Mathers C, Rebelo M et al. Cancer incidence and mortality worldwide: Sources, methods and major patterns in GLOBOCAN 2012. Int J Cancer 2014; 136: E359-E386.

2 Siegel R, Ma J, Zou Z, Jemal A. Cancer statistics, 2014. CA-Cancer J Clin 2014; 64: 9-29.

3 Levi F, Ferlay J, Galeone C, Lucchini F, Negri E, Boyle P et al. The changing pattern of kidney cancer incidence and mortality in Europe. BJU Int 2008; 101: 949-958.

4 Crumley S, Divatia M, Truong L, Shen S, Ayala A, Ro J. Renal cell carcinoma: Evolving and emerging subtypes. World J Clin Cases 2013; 1: 262.
5 Jonasch E, Futreal P, Davis I, Bailey S, Kim W, Brugarolas J et al. State of the science: an update on renal cell carcinoma. Mol Cancer Res 2012; 10: 859-880.

6 Axelson $H$, Johansson $M$. Renal stem cells and their implications for kidney cancer. Semin Cancer Biol 2013; 23: 56-61.

$7 \mathrm{Kim} \mathrm{K}$, Ihm H, Ro JY, Cho YM. High-level expression of stem cell marker CD133 in clear cell renal cell carcinoma with favorable prognosis. Oncol Lett 2011; 2: 1095-1100.

8 Gupta S, Rosenberg M. Do stem cells exist in the adult kidney? AM J Nephrol 2008; 28: 607-613.

9 Khan M, Czarnecka A, Helbrecht I, Bartnik E, Lian F, Szczylik C. Current approaches in identification and isolation of human renal cell carcinoma cancer stem cells. Stem Cell Res Ther 2015; 6: 178.

10 Mani S, Guo W, Liao M, Eaton E, Ayyanan A, Zhou A et al. The epithelialmesenchymal transition generates cells with properties of stem cells. Cell 2008; 133: 704-715.

11 Bussolati B, Bruno S, Grange C, Ferrando U, Camussi G. Identification of a tumor-initiating stem cell population in human renal carcinomas. FASEB $\mathrm{J}$ 2008; 22: 3696-3705.

12 Polyak K, Weinberg R. Transitions between epithelial and mesenchymal states: acquisition of malignant and stem cell traits. Nat Rev Cancer 2009; 9: 265-273.

13 Scheel C, Weinberg R. Cancer stem cells and epithelial-mesenchymal transition: concepts and molecular links. Semin Cancer Biol 2012; 22: 396-403. 
14 Thiery J, Acloque H, Huang R, Nieto M. Epithelial-mesenchymal transitions in development and disease. Cell 2009; 139: 871-890.

15 Singh A, Settleman J EMT, cancer stem cells and drug resistance: an emerging axis of evil in the war on cancer. Oncogene 2010; 29: 4741-4751.

16 Tiwari N, Gheldof A, Tatari M, Christofori G. EMT as the ultimate survival mechanism of cancer cells. Semin Cancer Biol 2012; 22: 194-207.

17 DiMeo T, Anderson K, Phadke P, Feng C, Perou C, Naber S et al. A novel lung metastasis signature links Wnt signaling with cancer cell self-renewal and epithelial-mesenchymal transition in basal-like breast cancer. Cancer Res 2009; 69: 5364-5373.

18 von Burstin J, Eser S, Paul M, Seidler B, Brandl M, Messer M et al. E-cadherin regulates metastasis of pancreatic cancer in vivo and is suppressed by a SNAIL/HDAC1/HDAC2 repressor complex. Gastroenterology 2009; 137: 361-371.e5.

19 He H, Yang X, Davidson A, Wu D, Marshall F, Chung L et al. Progressive epithelial to mesenchymal transitions in ARCaP E prostate cancer cells during xenograft tumor formation and metastasis. Prostate 2009; 70: 518-528.

20 Ishii K, Shimoda M, Sugiura T, Seki K, Takahashi M, Abe M et al. Involvement of epithelial-mesenchymal transition in adenoid cystic carcinoma metastasis. Int J Oncol 2011; 38: 921-931.

21 Micalizzi D, Ford H. Epithelial - mesenchymal transition in development and cancer. Future Oncol 2009; 5: 1129-1143.

22 Micalizzi D, Farabaugh S, Ford H. Epithelial-mesenchymal transition in cancer: parallels between normal development and tumor progression. J Mammary Gland Biol 2010; 15: 117-134.

23 Thiery J, Sleeman J. Complex networks orchestrate epithelial-mesenchymal transitions. Nat Rev Mol Cell Biol 2006; 7: 131-142.

24 Ye X, Weinberg R. Epithelial - mesenchymal plasticity: a central regulator of cancer progression. Trends Cell Biol 2015; 25: 675-686.

25 O'Mahony F, Faratian D, Varley J, Nanda J, Theodoulou M, Riddick A et al. The use of automated quantitative analysis to evaluate epithelial-tomesenchymal transition associated proteins in clear cell renal cell carcinoma. PLoS One 2012; 7: e31557.

26 Ho M, Tang S, Chuang M, Cha T, Li J, Sun G et al. TNF $\alpha$ induces epithelialmesenchymal transition of renal cell carcinoma cells via a GSK3 $\beta$ -dependent mechanism. Mol Cancer Res 2012; 10: 1109-1119.

27 Fang Y, Wei J, Cao J, Zhao H, Liao B, Qiu S et al. Protein expression of ZEB2 in renal cell carcinoma and its prognostic significance in patient survival. PLoS One 2013; 8: e62558.

28 Kouzarides T. Chromatin modifications and their function. Cell 2007; 128 : 693-705.

29 Greer E, Shi Y. Histone methylation: a dynamic mark in health, disease and inheritance. Nat Rev Genet 2012; 13: 343-357.

30 Martin C, Zhang Y. The diverse functions of histone lysine methylation. Nat Rev Mol Cell Biol 2005; 6: 838-849.

31 Kampranis S, Tsichlis P. Histone demethylases and cancer. Adv Cancer Res 2009; 102: 103-169.

32 Kim Y, Otterson G, Kratzke R, Coxon A, Kaye F. Differential specificity for binding of retinoblastoma binding protein 2 to RB, p107, and TATAbinding protein. Mol Cell Biol 1994; 14: 7256-7264.

33 Defeo-Jones D, Huang P, Jones R, Haskell K, Vuocolo G, Hanobik M et al. Cloning of cDNAs for cellular proteins that bind to the retinoblastoma gene product. Nature 1991; 352: 251-254.

34 Sellers W, Kaelin W. Role of the retinoblastoma protein in the pathogenesis of human cancer. J Clin Oncol 1997; 15: 3301-3312.
35 Benevolenskaya E, Murray H, Branton P, Young R, Kaelin W. Binding of pRB to the PHD protein RBP2 promotes cellular differentiation. Mol Cell 2005; 18: 623-635.

36 Mao S, Neale G, Goorha R. T-cell oncogene rhombotin-2 interacts with retinoblastoma-binding protein 2. Oncogene 1997; 14: 1531-1539.

37 Chan S. Retinoblastoma-binding Protein 2 (Rbp2) Potentiates Nuclear Hormone Receptor-mediated Transcription. J Biol Chem 2001; 276: 28402-28412

$38 \mathrm{Ge} \mathrm{Z}$, Li W, Wang N, Liu C, Zhu Q, Bjorkholm M et al. Chromatin remodeling: recruitment of histone demethylase RBP2 by Mad1 for transcriptional repression of a Myc target gene, telomerase reverse transcriptase. The FASEB J 2009; 24: 579-586.

39 Liefke R, Oswald F, Alvarado C, Ferres-Marco D, Mittler G, Rodriguez P et al. Histone demethylase KDM5A is an integral part of the core NotchRBP-J repressor complex. Gene Dev 2010; 24: 590-601.

40 Zeng J, Ge Z, Wang L, Li Q, Wang N, Bjorkholm M et al. The histone demethylase RBP2 is overexpressed in gastric cancer and its inhibition triggers senescence of cancer cells. Gastroenterology 2010; 138: 981-992.

41 Teng Y, Lee C, Li Y, Chen Y, Hsiao P, Chan M et al. Histone demethylase RBP2 promotes lung tumorigenesis and cancer metastasis. Cancer Res 2013; 73: 4711-4721.

42 Wang G, Song J, Wang Z, Dormann H, Casadio F, Li H et al. Haematopoietic malignancies caused by dysregulation of a chromatin-binding PHD finger. Nature 2009; 459: 847-851.

43 Sharma S, Lee D, Li B, Quinlan M, Takahashi F, Maheswaran S et al. A chromatin-mediated reversible drug-tolerant state in cancer cell subpopulations. Cell 2010; 141: 69-80.

44 Lin W, Cao J, Liu J, Beshiri M, Fujiwara Y, Francis J et al. Loss of the retinoblastoma binding protein 2 (RBP2) histone demethylase suppresses tumorigenesis in mice lacking Rb1 or Men1. P Natl Acad Sci 2011; 108: 13379-13386.

45 Blair L, Cao J, Zou M, Sayegh J, Yan Q. Epigenetic regulation by lysine demethylase 5 (KDM5) enzymes in cancer. Cancers 2011; 3: 1383-1404.

46 Wang S, Wang Y, Wu H, Hu L. RBP2 induces epithelial-mesenchymal transition in non-small cell lung cancer. PLoS One 2013; 8: e84735.

47 Mikami S, Mizuno R, Kosaka T, Saya H, Oya M, Okada Y. Prognostic significance of tnf-î \pm and cd44 expression in clear cell renal cell carcinomas: implication of cancer invasion, metastasis, and resistance to sunitinib treatment. J Urol 2014; 191: e306.

48 Belldegrun A. Renal cell carcinoma: prognostic factors and patient selection. Eur Urol Suppl 2007; 6: 477-483.

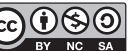

This work is licensed under a Creative Commons Attribution-NonCommercial-ShareAlike $\quad \mathbf{4 . 0}$ International License. The images or other third party material in this article are included in the article's Creative Commons license, unless indicated otherwise in the credit line; if the material is not included under the Creative Commons license, users will need to obtain permission from the license holder to reproduce the material. To view a copy of this license, visit http://creativecommons.org/licenses/by-nc-sa/4.0/ 